\title{
Impact of gestational age at PPROM on the short- term outcome of children born after extreme and prolonged preterm prelabor rupture of membranes in an experienced care center
}

\author{
HOCQ $C^{1}$, VAN GRAMBEZEN $B^{1,}$ BERNARD $P^{2}$, DEBAUCHE $C^{1}$ \\ ${ }^{1}$ Department of Neonatology, Cliniques Universitaires Saint Luc, Catholic University of Louvain, Belgium \\ ${ }^{2}$ Department of Obstetrics, Cliniques Universitaires Saint Luc, Catholic University of Louvain, Belgium \\ Autors Hocq C and Van Grambezen B contributed equally to the paper.
}

Corresponding author:

Hocq C

Department of Neonatology

Cliniques Universitaires Saint Luc

10 Avenue Hippocrate, 1200 Brussels, Belgium

Phone: +32 27649108

Fax: +3227649172

E-mail: catheline.hocq@uclouvain.be,benedicte.vangrambezen@uclouvain.be

\section{ABSTRACT}

Introduction. Survival of infants born after extreme PPROM (preterm prelabor rupture of membranes) has increased dramatically in the past 20 years, up to $90 \%$ in some tertiary neonatal centres, due to the progress in neonatal cardiorespiratory management.

Known risk factors of poor outcomes are lower gestational age at PPROM and prolonged and severe oligohydramnios.

Methods. We performed a retrospective study over a 6-year-period (2009-2015), including 14 pregnant women who experienced PPROM, before 25 weeks of gestation, with prolonged ( $>14$ days) and severe oligohydramnios (amniotic fluid index $<5$ ). Each live neonate was matched with a control patient who was born the same year, of the same gender, with the same gestational age (+/- 6 days) and who received treatment to induce fetal lung maturation at least 48 hours before birth.

Results. Live birth rate was 14/20 (70\%) and neonatal survival was 13/14 (93\%). Apgar scores at 5 and 10 minutes were lower in the PPROM group $(\mathrm{p}<0.01)$. Intubation was necessary for all babies with PPROM and for 5/13 (38\%) of the controls $(\mathrm{p}<0.01)$. In a subgroup analysis of the PPROM group, we found that all babies with PPROM $<20$ weeks presented refractory hypoxemia and required iNO (inhaled nitric oxide) administration compared to one in the PPROM group $>20$ weeks $(\mathrm{p}<0.01)$.

In all infants requiring iNO, the oxygena- tion index improved dramatically and rapidly with treatment.

We found no difference in the rate of bronchopulmonary dysplasia, necrotizing enterocolitis, retinopathy of prematurity or intraventricular hemorrhage.

Conclusion. PPROM before 20 weeks of gestation exposes the neonate to a high risk of refractory hypoxemia compared to PPROM after 20 weeks. The initial care management requires more aggressive treatment with administration of iNO in all of them.

After the initial period, the evolution of all babies born after PPROM is comparable to that of their controls.

Key words: preterm prelabor rupture of membranes, oligohydramnios, pulmonary hypertension, pulmonary hypoplasia.

\section{INTRODUCTION}

PPROM (preterm prelabor rupture of membranes) occurs in $3 \%$ of pregnancies (1-3) and represents one third of preterm deliveries. (2,3) Rupture of membranes before 24 weeks is much less common, affecting $0.4 \%$ of pregnancies. (4) Mortality for this condition has been very high historically: survival was 10 to $30 \%$ in the 90 's and progressively increased up to $70 \%$ in the year 2000. (1) In view of these poor outcomes, some centers are inclined to propose immediate TOP (termination of pregnancy) in cases of very early rupture of membranes, even nowadays. However, in some recent studies, survival rates can be as high as 70 to $90 \%$. $(1,5-7)$

In addition to the increased risk of mortality, these neonates are also at risk of several morbidities including hypoxemic respiratory failure, sepsis, limb contractures, potential neonatal asphyxia due to cord compression or placental abruption in addition to the risks of (extreme) prematurity.

Hypoxemic respiratory failure is attributable to pulmonary hypoplasia and arterial pulmonary hypertension. These two conditions appear in neonates after extreme PPROM associated with prolonged and severe oligohydramnios. The etiology of abnormal lung development is probably in part due to a dysregulation of pulmonary and vascular growth factors, which in turn is responsible for a decrease in alveolarisation. Abnormal pulmonary vasculature development is probably also implicated in the pathogenesis of this disease. (8) In these conditions, pulmonary pressure fails to decrease at birth and leads to persistent hypertension of the newborn (PPHN), which worsens hypoxemic respiratory failure.

Infection is a common complication of PPROM. The clinical chorioamnionitis rate is $30 \%$. (9) The most frequent pathogen detected in amniotic fluid is Ureaplasma urealyticum. This germ is known to induce an important inflammatory response in both the maternal and fetal membranes. (10) Inflammatory context is very often present in situations of extreme PPROM, linked to infection or not. This chronic inflammation can be deleterious for the fetus 
and is a risk factor for adverse outcome. Improvement of survival rate and decrease in morbidity became possible following the significant progress in antenatal management (corticosteroids and antibiotics essentially), in postnatal monitoring techniques (repeated cardiac scans) and in postnatal care management (surfactant administration, adjusted mechanical ventilation, use of inhaled nitric oxide). $(6,11)$ A first retrospective review was performed in our NICU (neonatal intensive care unit) between 2006 and 2008 and reported the outcomes of the highest risk babies who experienced severe (AFI,amniotic fluid index $<5$ ) and prolonged (latency time $>14$ days) PPROM before 25 weeks gestation. (1) The results published in 2009 were very promising (survival rate after admission to NICU was $78 \%$ ) and encouraged us to continue to take care of these newborns applying specific adjusted antenatal and postnatal care management.

We performed this second study to evaluate if the mortality and morbidity rates of these patients are still improving, with clinical experience in this field. For this purpose, we reviewed babies born after 2008, meeting the same inclusion criteria as in the first study. Our primary outcome was the rate of neonatal survival; secondary outcomes were maternal and neonatal morbidity.

\section{WHAT IS KNOWN AND WHAT IS NEW}

Mortality in infants born after extreme PPROM has decreased significantly in the past 20 years due to progress in neonatal cardiorespiratory management. This improvement was observed in our center, with survival rates increasing from $78 \%$ (1) to $93 \%$.

In our study, we confirmed the need for more aggressive care management in early PPROM (< 20 weeks), without increasing neonatal morbidity in these babies.

\section{MATERIALS AND METHODS}

This review is a retrospective study including pregnant women with PPROM who delivered in our obstetrical unit. The preterm neonates were admitted and treated in the level three neonatal intensive care unit of Cliniques Universitaires Saint-Luc, Brussels (Belgium) over a 6-year-period (September 2009 - August 2015).

This study was approved by the ethical committee.

Pregnancies were selected via the obstet- rical delivery data book which reports all deliveries occurring in our institution. All data were confirmed by reviewing the maternal medical file.

We included pregnant women presenting with the following confirmed criteria: PPROM before 25 weeks of pregnancy, confirmed by visualization of amniotic fluid with a speculum, by a PROM (IGFBP-1) or a positive Fern test or/and by visualization of oligohydramnios or anhydramnios on scan. In the event of doubt, diagnostic amnioinfusion was performed; duration of latency between PPROM and delivery more than 14 days; confirmed initial and persistent oligohydramnios $(\mathrm{AFI}<5)$ seen on initial scan and on weekly control scans. The exclusion criteria were as follows : associated fetal malformations and multiple pregnancy (they will be reviewed in a separate article).

Gestational age at rupture of membranes was obtained using the date of the last menstrual period, possibly corrected by ultrasound performed during the first trimester.

The management protocol in our institution for these pregnant women in the case of extreme preterm rupture of membranes is as follows: women are admitted to the maternal intensive care unit for initial work-up. Bed rest is prescribed and women receive antibiotics and tocolysis if required after exclusion of chorioamnionitis (amniocentesis is performed when technically possible). Antibiotics are initially given for 7 days, ampicillin or a second-generation cephalosporin is routinely used. Systematic blood analysis is performed every other day to detect infection. In case of doubt about chorioamnionitis, diagnostic amniocentesis is performed when technically possible. Antibiotic therapy is resumed if the systematic weekly vaginal swab is positive. Women remain hospitalized until delivery. Home discharge could be allowed with rehospitalization at the limit of viability. Induction of fetal lung maturation is performed between 24 and 26 weeks of gestation, after parental counseling.

These babies were identified and their medical data were used for analysis of neonatal outcomes.

Each neonate included in the study was matched with a control baby born the same year as the case index, of the same gestational age ( $+/-6$ days) and gender and who had received antenatal corticosteroids for fetal maturation at least 48 hours before birth, except for one control baby born at 34 weeks and 3 days of gestation without fetal maturation.

We reviewed maternal and fetal demo- graphic factors and analyzed maternal morbidity and neonatal outcomes in regard to respiratory, hemodynamic, infectious, digestive, neurological, ophthalmic and orthopedic problems.

Maternal sepsis is a maternal infection confirmed by a positive blood culture.

Chorioamnionitis is defined by histological criteria.

Bronchopulmonary dysplasia is defined as the need for oxygen at 28 days of life and 36 weeks of gestation.

Refractory hypoxemia is defined as the absence of saturation improvement after surfactant administration and mechanical ventilation.

PPHN is defined by clinical (pre and postductal saturation difference of more than $10 \%)$ and/or by echocardiographic criteria. Refractory hypotension is defined as persistent hypotension despite administration of 2 concomitant inotropic agents at maximal dose.

Early neonatal onset sepsis is defined by sepsis confirmed by a positive blood culture before 72 hours of life. Late-onset neonatal sepsis is an infection confirmed by a positive blood culture after 72 hours of life. NEC (necrotizing enterocolitis) was categorized following Bell's classification and only enterocolitis stage 2 or more was recorded as a digestive complication.

Intraventricular hemorrhage was graded according to Papile's staging. Only intraventricular hemorrhage $>2$ was reported. ROP (retinopathy of prematurity) was recorded as an ophthalmic complication if the patient had more than grade 2 retinopathy.

All blood gas analyses were done on arterial samples.

A subgroup analysis was performed for some outcomes, subdividing the results into two groups: the group of PPROM before 20 weeks and the group of PPROM after 20 weeks. The control group was also subdivided according to the criteria mentioned above. Comparisons were performed between each study subgroup and their control subgroup or between the 2 subgroups of PPROM.

Values are expressed in medians and interquartile range and were calculated using Microsoft excel for Mac 2011 version 14.5.9. Statistical analysis was performed using SPSS 20.0 software (SPSS, Chicago, Illinois). A Mann-Whitney test was used to compare the quantitative variables between studied groups and between each subgroup of PPROM with its control group or between the 2 subgroups of PPROM itself. A Fisher test was used to compare categorical values between the studied group 
and control group and between each subgroup of PPROM with its control group or between the 2 subgroups of PPROM itself. A $\mathrm{p}$ value less than 0.05 was considered to be statistically significant.

\section{RESULTS}

\section{Patients}

Flow diagram of study sample presented in figure 1 .

The live birth rate was $14 / 20(70 \%)$. One baby died at 11 hours of life from refractory hypoxemia and pulmonary hemorrhage. Autopsy was not performed. She was the youngest baby in our study population (birth at 25 weeks 5/7) and the smallest (birth weight of $680 \mathrm{~g}$ ). All the other 13 babies survived during the neonatal period and were discharged home. The survival rate after NICU admission was 13/14 (93\%).

Eight babies were born after PPROM occurring before 20 weeks (one of them died soon after birth) and six babies were born after PPROM occurring after 20 weeks of pregnancy. Neonatal demographic characteristics are shown in table 1.

For the babies with PPROM occurring before 20 weeks of gestation, the median gestational age at rupture was 18.4 (17.75-19.07) weeks; whereas it was 23 (22.78-23.21) weeks for those experiencing rupture after 20 weeks. There was no statistically significant difference for the median gestational age at birth whether rupture occurred before or after 20 weeks : 29.78 (28.21 - 30) weeks of GA (gestational age)

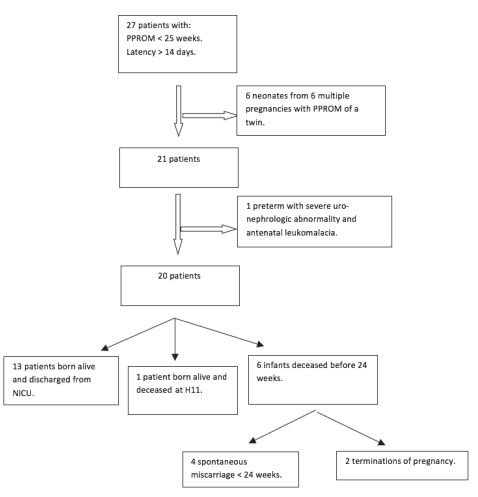

Figure 1. Flow diagram presenting patients with $P P R O M<25$ weeks, prolonged latency period and prolonged oligohydramnios.

AFI, amniotic fluid index; NICU, neonatal intensive care unit; PPROM, preterm prelabor rupture of membranes. versus 28.85 (27.85-29.85) weeks of GA ( $p$ $=0.79$ ). The median latency time between rupture and delivery increased significantly in the subgroup of PPROM before 20 weeks : 76 (68.5-84.5) days for babies born after PPROM before 20 weeks versus 37.5 (28-48.5) days for babies born following PPROM after 20 weeks $(\mathrm{p}<0.01)$.

\section{Maternal outcomes}

Three women presented with E. Coli sepsis. Two mothers were rehospitalized after discharge from the postnatal ward. One mother was rehospitalized 15 days after delivery caused by E.Coli chorioamnionitis with an infected retropelvic hematoma treated by intravenous antibiotic therapy. She was discharged from hospital after 5 days. The second patient was one of the three patients presenting with E.Coli sepsis; she was rehospitalized 50 days after delivery with an E. Coli tubo-ovarian abscess for which she underwent laparoscopy with abscess drainage and received intravenous antibiotic therapy. She was discharged from hospital after eight days.

There were no maternal infectious complications in the control group, in contrast with the study group. The difference between the two groups was not statistically significant.

\section{Neonatal outcomes}

\section{Respiratory outcome}

Neonatal data on invasive and non invasive ventilation are described in table 2.

Pneumothorax occurred in one patient in the PPROM group. It was a minor anterior pneumothorax with spontaneous resolution.

Rates of BPD (bronchopulmonary dysplasia) were identical between the PPROM group and control group. The three PPROM babies with BPD suffered PPROM before 20 weeks $(\mathrm{p}=0.19)$.

None of the patients needed oxygen at discharge.

The subgroup analysis showed that median duration of endotracheal ventilation was 5.8 (5-7.8) days for PPROM group $<20$ weeks, 4.1 (3.6-5.1) days for PPROM group $>20$ weeks, 0 (0-4.5) days for the control group of PPROM $<20$ weeks and $0.3(0$ 0.7 ) days for the control group of PPROM $>20$ weeks. The difference was significant when we compared the PPROM group and the control group ( $\mathrm{p}=0.01)$, PPROM subgroup $<20$ weeks and their own control subgroup $(\mathrm{p}=0.01)$ and $\mathrm{PPROM}<20$ weeks and PPROM $>20$ weeks. The differ- ence was borderline significant when we compared the PPROM group > 20 weeks and their own control group ( $\mathrm{p}=0.05)$.

The other subgroup analysis is presented in figures 2, 3 and 4 . These graphs show $\mathrm{pH}, \mathrm{pO} 2$ and pCO2 levels on admission to NICU as well as gestational age at weaning from any respiratory support.

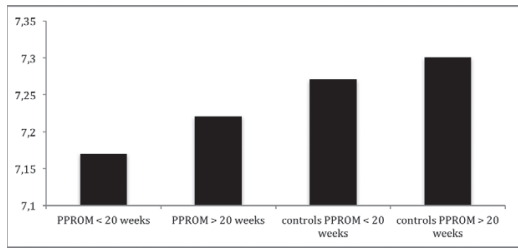

Figure 2. Arterial $\mathrm{pH}$ measured on admission to NICU (H2 of life). Difference is statistically significant between PPROM group $>20$ weeks and the control group $(p=0.01)$.

NICU, neonatal intensive care unit; PPROM, preterm prelabor rupture of membranes.

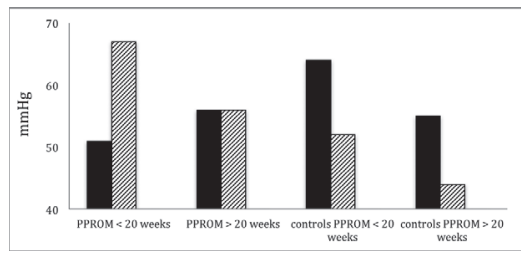

Figure 3. Mean arterial pO2 (filled column) and pCO2 (hatched column) measured on admission to NICU (H2 of life). Difference is close to being significant when comparing $\mathrm{pO} 2$ of PPROM $<20$ weeks with that of the controls ( $p=0.05$ ). All other comparisons between groups are not significant.

NICU, neonatal intensive care unit; PPROM, preterm prelabor rupture of membranes.

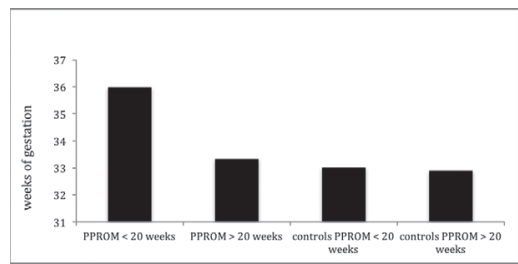

Figure 4. Mean gestational age at weaning from respiratory support in weeks.

Difference between groups is not significant. (Comparing

PPROM (preterm prelabor rupture of membranes) group $<20$ weeks with the PPROM group $>20$ weeks, $P=0.09$ ). 


\section{Hemodynamic outcomes}

8/13 patients from the PPROM group had refractory hypoxemia with $\mathrm{PPHN}$ requiring iNO (inhaled nitric oxide) therapy. All neonates presented with PPROM before 20 weeks except one $(p<0.01)$. Nitric oxide administration was started at a median time of 2.2 (1.7-3.3) hours of life and was sustained for a median of 3.3 (1.3-3.8) days. Improvement in oxygenation index was observed in the hours after commencing iNO (Figure 5).

All babies born after PPROM $<20$ weeks (7/7) received at least one inotropic agent (dobutamine or dopamine), 5/7 (71\%) of these patients received a combination of two inotropic drugs (dobutamine and dopamine). None of the control babies received inotropic agents $(\mathrm{p}<0.01)$.

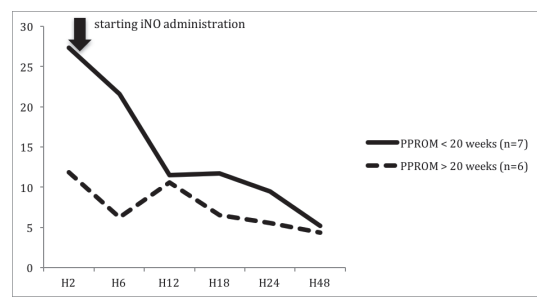

Figure 5. Mean oxygenation index of patients born with PPROM before 20 weeks and those born after 20 weeks.

iNO, inhaled nitric oxide; PPROM, preterm prelabor rupture of membranes.
3/7 patients with PPROM $<20$ weeks needed hydrocortisone for refractory hypotension. None of the babies born after PPROM > 20 weeks or the control babies needed hydrocortisone therapy $(\mathrm{p}=0.19)$. 2/13 babies in the PPROM group received AINS (antinflammatory nonsteroidal) therapy for PDA (patent ductus arteriosus), compared to one preterm in the control group. No babies required surgical ligation.

\section{Infectious outcomes}

Vaginal colonization and maternal bacteriological results are detailed in table 3 .

None of the babies presented early onset sepsis confirmed by a positive blood culture, but three babies in the PPROM group had clinical and biological signs compatible with early onset infection. All PPROM patients were given antibiotics for at least 3 days after birth.

Two patients in the PPROM group presented with late onset sepsis (2 CONS sepsis) during hospitalization, compared to four patients in the control group (3 CONS sepsis and 1 concomitant E. Coli and Klebsiella Pneumoniae sepsis). The difference between both groups was not statistically significant.

\section{Digestive outcomes}

One infant in the PPROM group had NEC stage III. None developed NEC in the control group.

None of the patients were discharged with tube feeding.

\section{Neurological and ophthalmological outcomes}

None of the neonates in the PPROM group or the control group developed neurological complications.

None of the patients presented with retinopathy of more than stage 2 .

\section{Orthopedic outcome}

Four preterm babies with PPROM were born with limb contractions ( 1 in the PPROM group $<20$ weeks and 3 in the PPROM group $>20$ weeks). They were all treated with physiotherapy and contractures resolved without need for surgery.

\section{Other outcomes}

We found no statistically significant differences regarding median length of stay between the PPROM group and their controls: 64 (49-80) versus 60 (55-68) days respectively $(\mathrm{P}=0.76)$. The median gestational age at discharge was 38.14 (37.14-39.28) weeks for PPROM patients and 38.14 (37.28-38.42) weeks for control infants ( $p$ $=0.55)$.

Table 1. Neonatal demographic factors, inclusive of the deceased patient and her control.

\begin{tabular}{|c|c|c|c|c|}
\hline & $\begin{array}{l}\text { PPROM born alive } \\
(\mathrm{n}=14)\end{array}$ & $\begin{array}{l}\text { Control infants } \\
(\mathrm{n}=14)\end{array}$ & p value (MW) & $\begin{array}{l}\text { PPROM deceased before viability } \\
(\mathrm{n}=6)\end{array}$ \\
\hline Antenatal corticosteroids & $14 / 14(100 \%)$ & $13 / 14(93 \%)^{\star *}$ & & $0 / 6(0 \%)$ \\
\hline $\begin{array}{l}\text { Latency between PPROM and } \\
\text { delivery (days) }\end{array}$ & $57.5(45-76.5)$ & l & & $19(18-25)$ \\
\hline Caesarean rate & $11 / 14(78 \%)$ & $10 / 14(71 \%)$ & & $2 / 6(33 \%)^{* * *}$ \\
\hline Gestational age at birth (weeks) & $29.86(28-30.28)$ & $29(27.61-30.11)$ & 0.84 & 22.07 \\
\hline Birth weight (grams) & $1375(995-1525)$ & $1047.5(820-1347.5)$ & 0.21 & $300(210-640)$ \\
\hline Apgar 1' & $4(1-5.75)$ & $5.5(5-7)$ & 0.07 & \\
\hline Apgar 5' & $5.5(5-7.75)$ & $8(8-9)$ & $<0.01^{*}$ & \\
\hline Apgar 10’ & $7(6-8.75)$ & $8.5(8-9)$ & $<0.01^{\star}$ & \\
\hline
\end{tabular}

*These values are statistically significant.

**The unique case control who did not receive antenatal corticosteroids was a late preterm baby born at 34 weeks and 3 days who was delivered within 24 hours after her mother was admitted to the obstetrical unit.

*** The 2 caesarean sections realized before viability were performed because of abdominal cerclage.

MW, mann-Withney test; PPROM, preterm prelabor rupture of membranes. 
Table 2. Neonatal data on invasive and non invasive ventilation, excluding the deceased patient and her control.

\begin{tabular}{llll}
\hline & PPROM $(\mathbf{n}=\mathbf{1 3})$ & Controls $(\mathbf{n}=\mathbf{1 3})$ & p value \\
\hline Intubation & $13 / 13(100 \%)$ & $5 / 13(38 \%)$ & $<0.01^{\star} \mathrm{F}$ \\
\hline Median time at intubation (minutes of life) & $19(10-25)$ & $90(36-94)$ & $<0.01^{\star} \mathrm{MW}$ \\
\hline Surfactant administration & $13 / 13(100 \%)$ & $6 / 13(46 \%)^{* *}$ & $<0.01^{\star} \mathrm{F}$ \\
\hline Duration of HFOV (days) & $5.3(3.9-5.7)$ & $0(0-0.7)$ & $<0.01^{\star} \mathrm{MW}$ \\
\hline Switch to conventional ventilation from HFOV & $6 / 13(46 \%)$ & $2 / 13(15 \%)$ & $=0.48 \mathrm{~F}$ \\
\hline Need of non invasive ventilation & $13 / 13(100 \%)$ & $11 / 13(85 \%)$ & $0.4 \mathrm{MW}$ \\
\hline Duration of non invasive ventilation (days) & $29(11-40)$ & $10(4-40)$ & \\
\hline
\end{tabular}

*these values are statistically significant.

${ }^{*} 1$ baby in the control group received surfactant without mechanical ventilation at 41 hours of life by INSURE technique.

F, Fisher test; HFOV, high frequency oscillatory ventilation; MW, Mann-Whitney test; PPROM, preterm prelabor rupture of membranes.

Table 3. Vaginal colonization and maternal bacteriological results.

\begin{tabular}{|c|c|c|}
\hline & PPROM born alive $(n=14)$ & PPROM deceased before viability $(n=6)$ \\
\hline Vaginal colonization & $\begin{array}{l}\text { E. Coli 8/14 }(57.1 \%) \\
\text { Enterococcus } 4 / 14(28.6 \%) \\
\text { Streptococcus agalactiae 3/14 (21.4\%) } \\
\text { Staphylococcus aureus 2/14 (14.3\%) } \\
\text { Klebsiella Pneumoniae 3/14 (21.4\%) } \\
\text { No pathogen colonization 2/14 (14.3\%) } \\
\text { ESBL bacteria 3/14 (21.4\%) }\end{array}$ & $\begin{array}{l}\text { E. Coli } 4 / 6(66 \%) \\
\text { Enterococcus } 3 / 6(50 \%) \\
\text { Streptococcus agalactiae }(20 \%) \\
\text { Staphylococcus aureus 1/6 (16.7\%) } \\
\text { Klebsiella Pneumoniae } 1 / 6(16.7 \%) \\
\text { Candida } 1 / 6(16,7 \%) \\
\text { No pathogen colonization } 1 / 6(16.7 \%) \\
\text { ESBL bacteria } 1 / 6(16.7 \%)\end{array}$ \\
\hline Antenatal antibiotherapy & $14 / 14(100 \%)$ & $4 / 6(66 \%)$ \\
\hline Placental anatomopathology & Histological chorioamnionitis 8/14 (57.1\%) & Histological chorioamnionitis $3 / 6(50 \%)$ \\
\hline Placental culture & $\begin{array}{l}6 \text { positive/ } 6 \\
\text { E. Coli }(2 x) \text {, Ureaplasma }(2 x) \text {, Klebsiella (1x), } \\
\text { Enterobacter }(1 \mathrm{x}) \text {. }\end{array}$ & $\begin{array}{l}3 \text { positive/3 } \\
\text { E. Coli }(2 \mathrm{x}) \text { with } 1 \text { E. Coli ESBL, Candida (1x). }\end{array}$ \\
\hline
\end{tabular}

ESBL, extended spectrum betalactamases; PPROM, preterm prelabor rupture of membranes.

\section{DISCUSSION}

Mid-trimester PPROM before viability is very rare. High mortality and morbidity rates among survivors are described in the literature, though some teams have described a dramatic improvement in mortality rate over the last 10 years. (1) These high morbidity and mortality rates are due to pulmonary hypoplasia and pulmonary arterial hypertension, which are more severe if the rupture occurs at an extreme gestational age and if the oligohydramnios is persistent and severe. (12) In our study, we reviewed this highest risk population of preterm babies who experienced PPROM before 25 weeks of gestational age with severe and prolonged oligohydramnios (AFI $<5$ - latency time $>14$ days).

We found a higher live birth rate after extreme premature rupture of membranes compared to some articles: $70 \%$ versus $40-$ $63 \%$ described in other studies. (13) This difference can be explained by our inclusion criteria, with a delay between rupture and delivery of at least 14 days. Therefore, all the babies born less than 14 days after PPROM were not taken into account. Comparing our study with that of Shah and Kluckow (6) and that of Everest et al., (4) who have similar inclusion criteria, we observe comparable results (69\% and 76\% of live births, respectively).

In our study, we demonstrated a survival rate of $93 \%$ for newborns admitted to NICU. This high survival rate is comparable to that of two recent studies based on similar inclusion criteria with a mortality rate below or equal to $10 \%$. $(6,13)$ However, several studies still report a very high mortality rate. There is still an important variation between studies, with a survival rate evaluated between 14 to $70 \%$. (14) It is of importance to note that the three studies, ours included, which reported a mortality rate less than $10 \%$, included patients with PPROM associated with persistent severe oligohydramnios.

Interestingly, our survival rate has been improving for the last 10 years. In 2009, our colleagues published a retrospective study based on the same inclusion cri- teria reporting the outcomes of infants born between 2006-2008, after extreme premature rupture of membranes. (1) The survival rate for infants admitted to NICU was $78 \%$. These results are comparable to those reported by Shah and Kluckow, (6) who showed an increase in survival rate comparing two successive periods. The increase in survival from $78 \%$ to $93 \%$ in our center may reflect the improvement in antenatal and postnatal care management, thanks to further experience in the management of these specific patients. But it may also reflect the impact of our first study on obstetric and neonatal staff attitudes, resulting in more optimistic parental counseling and more aggressive maternal and neonatal management. The increase in caesarean rate, from $53 \%$ in our first study to $79 \%$, may reflect this change in practice. The influence of the subjective caregivers' view on mortality and morbidity is mentioned by Azria et al. (15) They compared perinatal outcome after PPROM in two centers with different rates of TOP. Surprisingly, they demonstrated higher 
morbidity and mortality rates in the center that had a higher rate of termination of pregnancy. At the beginning of the study however, they expected the opposite, hypothesizing that the higher rate of TOP in one center would be a selection bias of the most favorable neonatal cases. This increase in survival can also reflect global improvement in neonatal care.

In this study we observed a great difference in the occurrence of refractory hypoxemia and pulmonary arterial hypertension between infants born after PPROM $<20$ weeks and those born after PPROM $>20$ weeks ( $100 \%$ versus $17 \%)$. These two conditions probably reflect a higher incidence of pulmonary hypoplasia in the group born after PPROM $<20$ weeks. No statistically significant difference was noted regarding gestational age at birth for both groups, reflecting a significantly longer latency time in the group of PPROM $<20$ weeks compared to the group of PPROM $>20$ weeks. This fact has already been reported by our colleagues who described that infants with clinical signs of pulmonary hypoplasia experienced a rupture at an earlier gestational age than those who did not have clinical signs of pulmonary hypoplasia (21.2 weeks versus 24 weeks $-p=0.07$ ). (1) These data reflect the importance of gestational age at which severe oligohydramnios appears, and the duration of severe oligohydramnios in the prediction of occurrence of pulmonary hypoplasia. This fact is reported by other authors who found that the occurrence of pulmonary arterial hypertension is inversely correlated with the gestational age at rupture $(1,13)$ which appears to be the best predictor of the occurrence of pulmonary hypoplasia in a meta-analysis performed in 2009. (12)

It is important to consider this marked difference in occurrence of pulmonary hypoplasia between PPROM $<20$ weeks and PPROM $>20$ weeks to offer the best possible parental counseling in the first place and secondly to anticipate very intensive care management of these babies. The pediatric team managing these babies at birth must be ready to perform prompt intubation and surfactant administration, in view of the poorer adaptation at birth of these babies, as demonstrated by the lower Apgar score in the subgroup of PPROM< 20 weeks. The team must also be ready to provide optimal adapted mechanical ventilation and to detect and treat pulmonary arterial hypertension promptly with iNO and administration of inotropics.

In this population of premature babies who suffer from pulmonary arterial hypertension, we observed a very rapid and positive response to iNO administration, as described previously by others. $(1,11)$ This demonstrates that at least part of the lung disease is reversible and may reflect that at least part of the disease is due to 'functional hypoplasia', so-called 'dry lung syndrome' (16) in addition to eventual irreversible pulmonary hypoplasia. Aikio et al. (17) demonstrated a transient NO deficiency in the first hours of life in patients born after PPROM and presenting with hypoxic respiratory failure, compared to those born after PPROM without hypoxic respiratory failure. They also showed different inflammatory patterns after birth between PPROM with and without hypoxic respiratory failure, with a less pronounced inflammatory response in the first hours of life for patients born after PPROM with hypoxic respiratory failure compared to those born after PPROM without hypoxic respiratory failure. (17) More studies are needed for a better understanding of the role of NO deficiency and the role of inflammatory patterns in pulmonary arterial hypertension developed by babies born after extreme PPROM.

While babies born after PPROM need a longer duration of invasive ventilation, especially those born after PPROM $<20$ weeks, there is no difference in the duration of non invasive ventilation and there is no higher occurrence of BPD at 36 weeks of gestation. None of these infants was discharged on oxygen support. These results show that after an initial need for more invasive respiratory and hemodynamic support, these infants evolve like their controls and this progression is dependent on gestational age. These results are also in part reported in the study by Verspyck et al. (18) After logistic regression analysis, this study showed that before 30 weeks of gestation, gestational age is the major independent factor associated with composite perinatal adverse outcome and with composite respiratory adverse outcome. However, authors found that PPROM was also an independent risk factor, but only for the composite respiratory adverse outcome.

In contrast to other studies, (18) we did not report any pneumothorax needing treatment among the PPROM patients. This result may be due to the use of HFOV (high frequency oscillatory ventilation) rather than conventional ventilation, the short period of mechanical ventilation and the experience acquired by our center.

Our study shows a rate of $57 \%$ of histological chorioamnionitis in the PPROM group. This rate of amnionitis remains very variable when reviewing the literature: the histological chorioamnionitis rate reported varies from $35 \%-40 \%$ in some studies $(19,20)$ to $66-75 \%$ in other studies. (15) In this study, all the placentas were analyzed for histological chorioamnionitis. In many other articles, there is no specification about the manner in which the diagnosis of chorioamnionitis was obtained.

None of the babies had proven early onset sepsis based on a positive blood culture and only a few patients showed clinical and biological signs of infection. This can be explained by close monitoring of maternal infection signs and maternal antibiotic administration. Some studies suggested a link between the interval PPROM-delivery and the risk of chorioamnionitis and neonatal infection with increased infectious risk correlated with increase in latency time, but other reports did not support this association. (21)

The babies born after PPROM do not have a higher risk of late-onset infection in comparison with control babies. It remained difficult to compare the infectious risk for these babies as most authors refer to sepsis, but do not specify if it is early-onset sepsis or late-onset sepsis.

Analyzing other morbidities, we found an increase in orthopedic morbidities in the group of PPROM, including limb retraction and positional syndrome. These complications were transitional as none of the newborns required surgery and all demonstrated good evolution, using physiotherapy only.

We found no increase in digestive complications, like NEC or feeding intolerance. There was no increase in ROP, or intraventricular hemorrhage in the studied group of PPROM compared to the controls. These data appear to be very encouraging, showing that in the event of extreme PPROM the principal morbidities in addition to prematurity itself are pulmonary and hemodynamic problems, at least short term. An analysis of the longer term neurological complications in this population is needed to be sure that the evolution of this population is not worse compared to the control population. However, data from our colleagues' follow-up study of their initial population at two years of age, (22) did not reveal a worse neurological outcome. In contrast, pulmonary long-term outcomes showed an increase in rehospitalization for respiratory problems during the two first years of age and an increase in the need for treatment with inhaled bronchodilators and inhaled corticosteroids.

A maternal mortality rate of $1 \%$ is described in the literature. (20) In this review, we had no maternal deaths and the number of complications observed was 
not significantly increased in comparison to those presented by the mothers in the control group. However, three cases of invasive infection with Gram negative bacteria were found requiring prolonged antibiotic therapy for the mothers. Unfortunately, we noted that around $20 \%$ of the women had multiresistant bacteria. This can be explained by multiple antibiotics and a prolonged hospital stay. This shows the importance of selective antibiotics, i.e., avoidance of broad spectrum antibiotics, since the use of antibiotics after PPROM is a well-known protective factor. (10)

Our study has numerous limitations. Firstly, this is a retrospective study. Secondly, only a small number of patients was included. These two characteristics may explain why we did not show more significant results or demonstrate more complications in the whole group of PPROM and particularly in the subgroup analysis of PPROM. However, the fact that our results are at least comparable and even better than those of the first study conducted in our centre, strengthens our results. Another limitation can be found in the control group, as prematurity was due to other etiologies, such as chorioamnionitis or preeclampsia, which are associated with specific morbidity. However, we tried to reduce selection bias by selecting infants of the same gestational age, the same gender and the same degree of fetal lung maturation. To our knowledge, these last criteria have never been taken into account in other studies on the topic.

A prospective multicenter study should be conducted to strengthen these shortterm results. Providing a follow-up to this population at high risk of morbidity is also essential to evaluate long- term complications, not only at two years, but throughout infancy, until adulthood.

\section{CONCLUSIONS}

This study demonstrates an increase in survival rate of babies born after PPROM $<25$ weeks with prolonged and severe oligohydramnios in a single centre in the past 10 years and demonstrates the importance of specific management and practical ex- perience.

This study shows that rupture before 20 weeks results in worse initial respiratory and hemodynamic status, with arterial pulmonary hypertension in all cases. This arterial hypertension is at least in part reversible, with an excellent response to iNO administration. After the first days of life, these babies have a similar evolution compared to control babies; the pulmonary prognosis in the neonatal unit is related to gestational age.

This study does not reveal any other significant irreversible morbidity, in particular no increase in neurological morbidity in the neonatal period.

\section{ACKNOWLEDGEMENTS}

We would like to acknowledge the contribution of Damien Moerman, physiotherapist in the pediatric intensive care unit in Cliniques Universitaires Saint-Luc, Brussels, for statistical assistance.

\section{REFERENCES}

1. Williams O, Hutchings G, Debiève F, Debauche C. Contemporary neonatal outcome following rupture of membranes prior to 25 weeks with prolonged oligohydramnios. Early Hum Dev 2009;85(5): 273-7.

2. Goldenberg RL, Culhane JF, Lams JD, Romero R. Epidemiology and causes of preterm birth. Lancet 2008;371:75-84.

3. Kenyon SL, Taylor DJ, Tarnow-Mordi W; ORACLE Collaborative Group. Broad-spectrum antibiotics for preterm, prelabour rupture of fetal membranes: the ORACLE I randomized trial. Lancet 2001; 357(9261):979-88.

4. Everest NJ, Jacobs SE, Davis PG, Begg L, Rogerson S. Outcomes following prolonged preterm premature rupture of membranes. Arch Dis Child Fetal Neonatal Ed 2008;93:207-11.

5. Brumbaugh JE, Colaizy TT, Nuangchamnong N, O’Brien EA, Fleener DK, Rijhsinghani A, Klein JM. Neonatal survival after prolonged preterm premature rupture of membranes before 24 weeks of gestation. Obstet and Gynecol 2014;124:992-8.

6. Shah DM, Kluckow M. Early functional echocardiogram and inhaled nitric oxide: usefulness in managing neonates born following extreme preterm premature rupture of membranes (PPROM). J Paediatr Child Health 2011;47(6):340-5.

7. Welzing L, Bagci S, Abramian A, Bartmann P, Berg C, Mueller A. CPAP combined with inhaled nitric oxide for treatment of lung hypoplasia and persistent foetal circulation due to prolonged PPROM. Early Hum Dev 2011;87(1):17-20.

8. Williams O, Hutchings G, Hubinont C, Debauche C, Greenough A. Pulmonary effects of prolonged oligohydramnios following midtrimester rupture of the membranes-antenatal and postnatal management. Neonatology 2012;101(2):83-90.

9. Kacerovsky M, Vrbacky F, Kutova R, Pliskova L, Andrys C, Musilova I, et al. Cervical microbiota in women with preterm prelabor rupture of membranes. PloS One 2015;10(5):e0126884.

10. Gomez R, Romero R, Nien JK, Medina L, Carstens M, Kim YZ, et al. Antibiotic administration to patients with preterm premature rupture of membranes does not eradicate intra-amniotic infection. J Matern Fetal Neonatal Med 2007;20(2):167-73.

11. de Waal K, Kluckow M. Prolonged rupture of membranes and pulmonary hypoplasia in very preterm infants: pathophysiology and guided treatment. J Pediatr 2015;166(5):1113-20.

12. van Teeffelen AS, van der Ham DP, Oei SG, Porath MM, Willekes C, Mal BW. The accuracy of clinical parameters in the prediction of perinatal pulmonary hypoplasia secondary to midtrimester prelabour rupture of fetal membranes: a meta-analysis. Eur J Obstet Gynecol Reprod Biol 2010;148(1):3-12.

13. Homer L, Bernard C, Collet M. Management and outcomes of pregnancies complicated by preterm premature rupture of membranes before 26 weeks of gestation. Gynecol Obstet Fert 2014;42(2):71-7.

14. van der Heyden JL, van der Ham DP, van Kulijk S, Notten KJ, Janssen T, Nijhuis JG, et al. Outcome of pregnancies with preterm prelabor rupture of membranes before 27 weeks' gestation : a retrospective cohort study. Eur J Obstet Gynecol Reprod Biol 2013;170 (1):125-30.

15. Azria E, Anselem O, Schmitz T, Tsatsaris V, Senat MV, Goffinet F. Comparison of perinatal outcome after pre-viable prelabour rupture of membranes in two centers with different rates of termination of pregnancy. BJOG 2012;119(4):449-57.

16. Sehgal A, Francis JV, Ang H, Tan K. Dry lung syndrome: a distinct clinical entity. Indian J Pediatr 2010;77(9):1029-31. 
17. Aikio O, Metsola J, Vuolteenaho R, Perhomaa M, Hallman M. Transient defect in nitric oxide generation after rupture of fetal membranes and responsiveness to inhaled nitric oxide in very preterm infants with hypoxic respiratory failure. J Pediatr 2012;161(3):397403.

18. Verspyck E, Bisson V, Roman H, Marret S. Adverse respiratory outcome after premature rupture of membranes before viability. Acta Paediatr 2014;103(3):256-61.

19. Waters TP, Mercer BM. The management of preterm premature rupture of the membranes near the limit of fetal viability. Am J Obstet Gynecol 2009;201(3):230-40.

20. Newman DE, Paamoni-Keren O, Press F, Wiznitzer A, Mazor M, Sheiner E. Neonatal outcome in preterm deliveries between 23 and 27 weeks' gestation with and without preterm premature rupture of membranes. Arch Gynecol Obstet 2009;280(1):7-11.

21. Drassinover D, Friedman AM, Obican SG, Levin H, Gyamfi-Bannerman C. Prolonged latency of preterm premature rupture of membranes and risk of neonatal sepsis. Am J Obstet Gynecol 2016 Jun;214(6):743.e1-6.

22. Williams O, Michel B, Hutchings G; Debauche C, Hubinont C. Two-year neonatal outcome following PPROM prior to 25 weeks with a prolonged period of oligohydramnios. Early Hum Dev 2012;88(8):657-61. 